\title{
La bifurcation infrastructurelle
}

Comment compléter les théories urbaines sur les réseaux techniques urbains?

Infrastructural bifurcation. How to enrich the urban theories dealing with urban technical networks?

\section{Daniel Florentin}

\section{(2) OpenEdition}

\section{Journals}

Édition électronique

URL : https://journals.openedition.org/ress/4060

DOI : $10.4000 /$ ress. 4060

ISSN : $1663-4446$

Éditeur

Librairie Droz

Édition imprimée

Date de publication : 25 mai 2018

Pagination : 241-262

ISSN : 0048-8046

\section{Référence électronique}

Daniel Florentin, «La bifurcation infrastructurelle», Revue européenne des sciences sociales [En ligne], 56-1 | 2018, mis en ligne le 25 mai 2021, consulté le 07 janvier 2022. URL : http://

journals.openedition.org/ress/4060 ; DOI : https://doi.org/10.4000/ress.4060 


\title{
LA BIFURCATION INFRASTRUCTURELLE COMMENT COMPLÉTER LES THÉORIES URBAINES SUR LES RÉSEAUX TECHNIQUES URBAINS?
}

DANIEL FLORENTIN

\author{
ISIGE - Mines Paris Tech, PSL Research University \\ daniel.florentin@mines-paristech.fr
}

\begin{abstract}
Résumé. Depuis une vingtaine d'années, on assiste, principalement en Europe, à une transformation majeure des modalités de fonctionnement et de gestion des réseaux techniques urbains (eau, chauffage, gaz, électricité). Cette transformation se manifeste, entre autres, par des changements de régime de demande et une baisse de consommation des fluides circulant dans les réseaux, à l'opposé des dynamiques historiques d'évolution des réseaux et en contradiction avec le modèle économique sur lequel ils ont été conçus. Pareille transformation se trouve en fait à la confluence d'interrogations émanant des opérateurs industriels et de questions académiques émergentes, et est même devenue un objectif de politique publique dans le cadre des politiques de sobriété énergétique. Elle signe le premier pas d'une bifurcation infrastructurelle, dont les modalités de fonctionnement constituent le cœur de ce texte.
\end{abstract}

Mots-clés: bifurcation, eau, Europe, opérateur, régime de demande, réseaux techniques urbains.

\begin{abstract}
Over the last couple of decades, urban technical systems (water, gas, power and heating systems) have experienced a massive transformation. Such a change is predominantly affecting European cities and translates into shrinking consumption levels and consequently changing demand regimes that are contradicting historical evolution dynamics and traditional business models of networked infrastructures. This modification lies at the convergence of a larger questioning burgeoning both within industrial operators and academic circles: it has even become the core of public policies of low-carbon and energy transitions. The paper argues this should be conceived as the first sign of an infrastructural bifurcation, whose modalities and functioning are further detailed in the text.
\end{abstract}

Keywords: bifurcation, demand regime, Europe, urban networked infrastructures, utilities, water. 


\section{INTRODUCTION Des modèles d'évolution du réseau incomplets}

Dans la grammaire de l'aménagement urbain, la figure du réseau technique joue un rôle central de conjonction et de transformation. Elle alterne, pour reprendre les termes de Jean-Marc Offner (1993) entre la «machine circulatoire», qui permet l'approvisionnement d'une ville en fluides et services essentiels à son fonctionnement, et «l'instrument de territorialisation», qui voit le réseau profondément changer l'organisation du territoire, en le hiérarchisant autour de nœuds et de lignes de circulation. Cette machine circulatoire ne se limite pas à sa seule dimension matérielle et technique mais englobe aussi la firme exploitant le réseau, l'usager et le territoire desservis. Cette approche reprend l'ambition affirmée par Sylvy Jaglin (2005) d’avoir une conception large du réseau, portant sur le service et ceux qui le mettent en ouvre, et pas seulement sur le tuyau ou le câble. Le réseau ainsi compris a longtemps été inextricablement lié à un principe de fonctionnement, celui d'une croissance continue de la demande, qui en a assuré la pérennité aussi bien technique qu'économique. Cette croissance est même consubstantielle aux grands réseaux techniques (Kornwachs, 1993; Offner, 1996), puisque l'efficacité du réseau est censée croître avec sa taille et que la demande est considérée comme inextinguible (Coutard, 20I0a). Le (grand) réseau technique se nourrit donc assez largement d'une forme d'idéalisation de la croissance et d'un système de consommation non borné (Coutard, 20Iob) : le réseau a été en quelque sorte dopé à la croissance, ce qui lui a permis d’asseoir sa stabilité économique et territoriale.

Cette machine semble cependant avoir vu son mécanisme quelque peu se gripper, sous l'effet concomitant d'un discours, remettant en cause la figure du réseau et ses bénéfices par certains acteurs urbains (décrits notamment dans leurs travaux sur le post-réseau par Coutard et Rutherford, 20I6) et d'un processus, observable essentiellement en Europe, consistant dans la baisse de consommation des fluides circulant dans les réseaux d'eau ou d'énergie (Barraqué et al., 20I i ; Florentin, 2015). Ces baisses, dont les facteurs explicatifs sont multiples, témoignent de l'émergence de nouveaux régimes de demande, situés à l'opposé des dynamiques historiques d'évolution des réseaux et en 
contradiction avec le modèle économique sur lequel ils ont été conçus, générant notamment de nouveaux coûts échoués.

Ces nouveaux régimes de demande altèrent les fonctionnalités techniques des réseaux (Moss, 2008) et mettent plus généralement à mal le fonctionnement traditionnel des réseaux techniques, menaçant, à (long) terme, l'existence des réseaux concernés par ces processus. Ils appellent de possibles adaptations et ouvrent une phase nouvelle dans l'évolution des réseaux, qui n’avait jusqu'ici pas été envisagée. Cette transformation des régimes d’infrastructure urbaine (Monstadt, 2009) est assez inédite et correspond en fait à un impensé des modèles développés jusqu'ici sur l'évolution des réseaux (Offner, 1993). Les transformations affectant les réseaux techniques urbains étaient souvent pensées comme la résultante d'une augmentation de la production ou de la consommation $^{1}$ (Garrison, I991 ; Offner, I993 ; Curien, 2000), et presque jamais comme le fruit possible d'un retournement des dynamiques de la demande.

En s'appuyant sur l'analyse approfondie de deux opérateurs de réseaux techniques urbains représentatifs de ces nouveaux régimes de demande (à Magdeburg et à Séville) et sur leur mise en perspective dans la littérature scientifique et technique, cet article forge l'hypothèse que la transformation profonde qui affecte une partie des réseaux techniques urbains peut être qualifiée de bifurcation infrastructurelle, à savoir un changement radical d'orientation dans le fonctionnement des systèmes techniques urbains. Ces changements modifient également la façon d'envisager les réseaux et de compléter les modèles d'évolution qui avaient été tracés jusqu'ici.

Après un rapide cadrage de la notion de bifurcation infrastructurelle (I), ce texte analyse l'émergence d'un nouveau régime de demande dans les réseaux (2). Il synthétise ensuite les transformations opérées par les firmes de réseaux pour s'adapter à ces nouveaux régimes (3), pour voir dans quelle mesure on peut compléter les modèles d'interprétation et d'évolution des réseaux (4).

I «Shifts in network structure are driven by changes in the means or technologies used for transactions, as well as by increased production and consumption» (Garrison, 1991, p.6). 


\section{DE LA NOTION DE BIFURCATION INFRASTRUCTURELLE}

Le terme de bifurcation peut avoir plusieurs acceptions. Il est ici compris dans son sens courant, rattaché aux questions routières, qui voit une route se diviser en deux. Il sert à décrire un changement radical d'orientation dans le fonctionnement des systèmes techniques urbains.

Dans son modèle de croissance des réseaux, Offner montrait qu'un réseau (technique urbain) était constitué de cinq caractéristiques: une morphologie, une infrastructure (le réseau-support), une fonctionnalité, un mode de régulation et une territorialité (Offner, 1993). Dans son modèle, il rappelait qu’en raison de la forte matérialité des réseaux, leurs évolutions étaient souvent lentes et ne modifiaient qu'une ou deux de ces caractéristiques sur une période donnée.

L'hypothèse que je forme est que cette modification, dans le cadre de nouveaux régimes de demande, peut être plus profonde et est sans doute d'un genre inédit, par son ampleur et sa diffusion. Pour en prendre la mesure, j'emprunte à Stephen Graham et Simon Marvin une partie de leur démarche à défaut d'en suivre l'ensemble des conclusions, dans ce qu'ils présentaient comme une thèse dans leur ouvrage Splintering Urbanism (200I). Selon eux, une partie des réseaux est en train de se développer de façon parallèle aux réseaux traditionnels, créant de nouveaux lieux de services différenciés (les «premium network spaces»); cette dynamique viendrait entériner et renforcer la fragmentation du tissu social et de la fabrique matérielle de la ville. De la même façon, une autre trajectoire assez nette est en train d'émerger pour de nombreux opérateurs de réseau dans les pays occidentaux (et notamment européens), qui remet en cause leur modèle de fonctionnement et les modalités de gestion des espaces urbains desservis. Pareille évolution pourrait être qualifiée de «flipside of the splintering urbanism narrative» (Moss, 2008). Cette trajectoire émergente ne dit pas l'évolution de l'ensemble des réseaux techniques urbains et connaît de nombreuses variations selon les contextes locaux. Toutefois, elle semble marquer de façon assez profonde une part non négligeable des mondes techniques urbains, notamment européens. C'est la raison pour laquelle je qualifie cette évolution de bifurcation infrastructurelle. 
La notion de bifurcation a été développée dans le champ des recherches sur les villes en déclin (Beauregard, 2006; Baron et al., 20ı). L’analyse des évolutions de ce que la littérature anglo-saxonne nomme des shrinking cities passe en particulier par l'idée que ce processus de déclin n’est pas un réajustement temporaire, mais bien une nouvelle normalité (Hannemann, 2003). La bifurcation est donc à comprendre comme un changement structurel du système (Djament-Tran et Reghezza-Zitt, 20ı2) : «La bifurcation ne peut signifier un simple retour aux états antérieurs. [...] La bifurcation signifie en revanche que les équilibres entre les éléments du système sont affectés par le changement voire la disparition de certains d'entre eux » (Baron et al., 20I0, p. 33)

Cette transition impliquerait des changements profonds, non pas simplement des mitigation strategies, mais des adaptation strategies, pour reprendre la distinction classique faite à propos des stratégies pour lutter contre le changement climatique, reprise et conceptualisée notamment par Biesbroek, Swart et Van der Knaap (2009) ${ }^{2}$. Elle ne se limiterait pas à de simples changements techniques, comme ce «mythe opératoire de l'aménagement urbain »(Zepf et al., 2008, p. I3 I) que serait la simple transition d'un système marqué par les grands systèmes techniques à des systèmes plus décentralisés. Elle ne relèverait pas non plus de la croyance quasi religieuse dans les vertus du smart grid (réseau dit intelligent), telle que la développent certaines publications professionnelles (UK Water Industry Research, 20ı0) ${ }^{3}$. On assisterait bien plutôt à ce que certains auteurs ont déjà qualifié de changement de paradigme (Kluge et Scheele, 2003 ; Zepf et al., 2008), marqué par l'émergence de nouveaux modèles techniques, professionnels, économiques et territoriaux.

2 D'après eux, les stratégies d'adaptation relèvent d'une approche plus systémique et réactive, là où les stratégies d'atténuation restent souvent plus mono-disciplinaires et proactives.

3 Le rapport de la UK Water Industry Research espère un saut technologique en frisant la caricature, par la répétition du terme «smart» à plus de 80 reprises en moins de 30 pages dans l'un des scénarios développés. 
Les réseaux étant essentiellement une «grammaire des forces et des rapports de forces » (Callon et Ferrary, 2006), on peut considérer que, derrière cette bifurcation infrastructurelle, se jouent des enjeux de pouvoir sur les modes d'approvisionnement des services urbains, pour chercher une voie tierce dépassant les logiques de déconnexion de certains habitants (Lopez, 20I4) et de fragmentation socio-spatiale aggravée par les réseaux (Graham et Marvin, 200I).

\section{DE LA CROISSANCE COMME MOTEUR À UN NOUVEAU RÉGIME DE DEMANDE}

Cette bifurcation repose sur un changement de régime de demande, d'un réseau à régime croissant à l'émergence d'un réseau à régime décroissant.

\section{I. LA CROISSANCE COMME MOTEUR DU RÉSEAU}

Cette logique de croissance à tout prix a été portée en particulier par l'absence de contraintes énergétiques qui a prévalu pendant plusieurs décennies (Owens, 1986), limitant de fait les considérations sur les questions d'efficience du système existant, qu'il s'agisse d'un réseau d'eau, de chauffage ou de gaz. La logique d'extension inhérente au réseau a ainsi joué également un rôle de moteur dans l'étalement urbain, quand bien même celui-ci n'était pas entièrement compatible avec un approvisionnement collectif (Owens, 1986).

Cette attraction pour la croissance est portée par un imaginaire et des pratiques professionnelles : les planifications urbaine et infrastructurelle sont ainsi marquées par des décennies de programmes et de plans élaborés à l'aune de la seule croissance (Moss, 2008; Monstadt, 2009). Elle s'appuie également sur quelques éléments d’analyse économique insuffisamment poussés, reposant sur le principe largement répandu dans les sociétés occidentales qui veut qu'une consommation croissante mène à un bien-être plus élevé (Jackson, 2005). Certaines études ont ainsi pu montrer le lien entre la croissance du revenu par habitant et la croissance de la demande en eau et énergie (Villarin et Camarillo, 2012). Le réseau se nourrit ainsi doublement de la croissance de l'urbanisation et de l'enrichissement progressif des urbains et des sociétés occidentales toujours plus urbaines. On retrouve cette idée énoncée avec force 
dès le XIX ${ }^{e}$ siècle, où la croissance continue des consommations et du réseau est annoncée non seulement comme un objectif économique mais aussi comme un programme culturel. C'est ce qu'on peut lire notamment dans ces pages étonnantes de l'ouvrage de l'architecte autrichien Adolf Loos, qui, dans Die Plumber, rappelle que «seul le peuple qui approchera les Anglais pour la consommation d'eau sera capable de rivaliser avec eux dans l'ordre économique [...]. L'augmentation de la consommation d'eau est une de nos tâches culturelles les plus pressantes » (Loos, I898, p. 55). On ne saurait mieux dire l'ambition civilisatrice qu'est censée porter la croissance de la consommation et son vecteur, le réseau technique.

Cependant, ce lien mécanique du réseau à la croissance de la demande a été modifié au cours des vingt dernières années, transformant par ce biais le fonctionnement du réseau.

\subsection{CRISE DU GRAND RÉSEAU ET RÉSEAU À RÉGIME DÉCROISSANT}

Depuis le début des années 1990, dans la plupart des villes européennes, un phénomène inédit a commencé à être observé, avec plus ou moins d'intensité selon les cas, mais concourant au processus de crise des grands réseaux (Coutard, 20ı): la baisse des consommations de certains réseaux, et en particulier des réseaux d'eau. Celle-ci s'est développée sous l'effet conjoint de processus de désindustrialisation (et en particulier l'érosion progressive de secteurs très aquavores ou énergivores), d’efficacité accrue de l'équipement électroménager domestique (ou de l'isolation pour le chauffage urbain), des augmentations tarifaires et autres dispositifs de gestion de la demande. Les cas de Séville en ce qui concerne l'eau et de Magdeburg pour le chauffage urbain en offrent une illustration assez classique, avec une baisse assez importante sur le long terme, quoique pas toujours linéaire (Figures I et 2). Les effets négatifs de ces baisses sont multiples et ont été déjà en partie documentés (Moss, 2008 ; Florentin, 20I5) : élévation des risques d'hygiène liés à la stagnation de l'eau dans les conduites, déperdition aggravée de chaleur, surcoûts d'entretien, baisse des recettes sachant que la facturation est principalement volumétrique. 
Figure I. Baisse de la consommation de chauffage urbain à Magdeburg

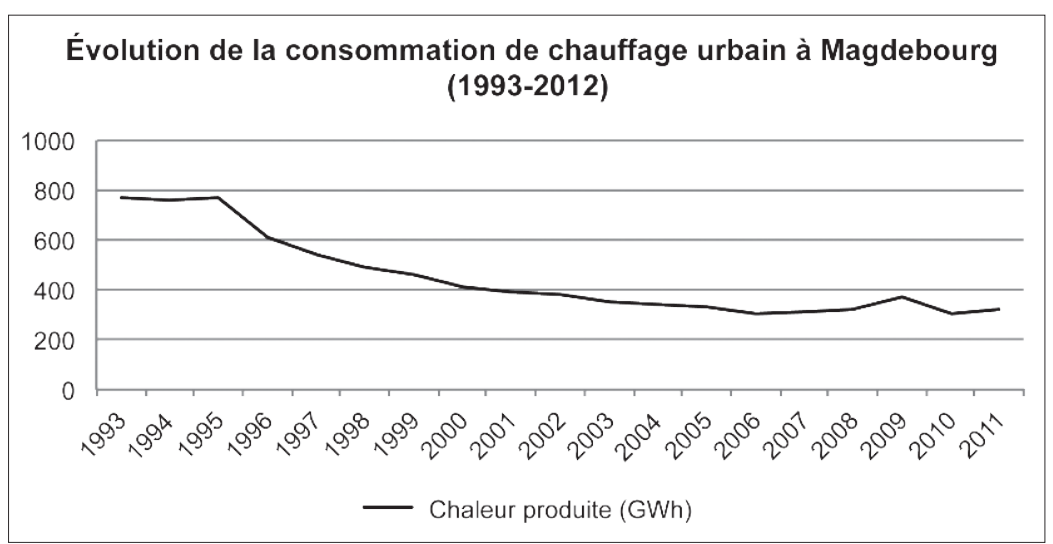

Source: données SWM.

Figure 2. Séville et l'eau : augmentation de la population, baisse de la consommation

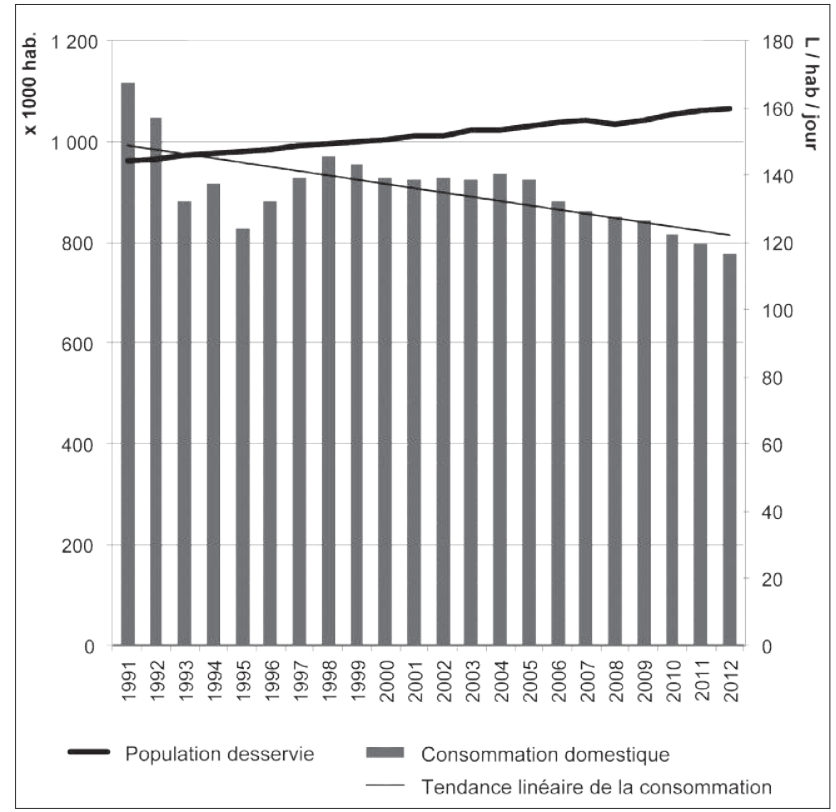

Source: données EMASESA. 
Ces baisses sont mêmes devenues récemment un objectif de politique publique dans le cadre des politiques de sobriété énergétique ou dans les programmes portant sur une éventuelle transition énergétique: les changements de demande sont ainsi devenus des objectifs politiques et sociaux, justifiant l'idée que se modifie un régime de demande.

Si les réflexions sur ces changements sont encore balbutiantes dans le secteur de l'énergie ou dans certains services en réseau, elles font l'objet d'études plus fournies dans le secteur de l'eau (Barraqué et al., 20II; Euzen, 2004; Pflieger, 2009) et d'un intérêt des opérateurs plus fortement affirmé, sans doute en raison de l'ampleur du phénomène, du caractère vieillissant des réseaux et de la difficulté à les maintenir en bon état (Bouleau, 20I I), qui vient heurter la vision d'un système à croissance infinie. Même s'il concerne progressivement tous les réseaux techniques, ce nouveau régime de demande est principalement visible et stabilisé dans le domaine de l'eau.

Pourtant, ce phénomène de changement de modèle économique lié à un changement de régime de demande semble prendre de court la plupart des opérateurs. Tout du moins note-t-on une relative impréparation aux effets aussi bien techniques, économiques, sociaux que territoriaux qu'il pourrait avoir. Le processus n'est reconnu que depuis peu par les opérateurs comme un processus pérenne et multidimensionnel (Koziol, 2008 pour le cas allemand; Albiol Omella et Agulló Amorós, 20I4 pour la première étude y faisant référence en Espagne). Cette impréparation peut sembler paradoxale au vu des nombreuses campagnes incitant à des consommations plus sobres développées par les opérateurs eux-mêmes depuis plus d'une dizaine d’années (Figure 3). Elle témoigne sans doute d'un changement de pratiques et de cultures professionnelles qui n'est pas encore pleinement abouti pour s'adapter aux enjeux du surdimensionnement. 
Figure 3. Campagne des années 2000: «aidez-nous à éviter que l'eau ne devienne un luxe»

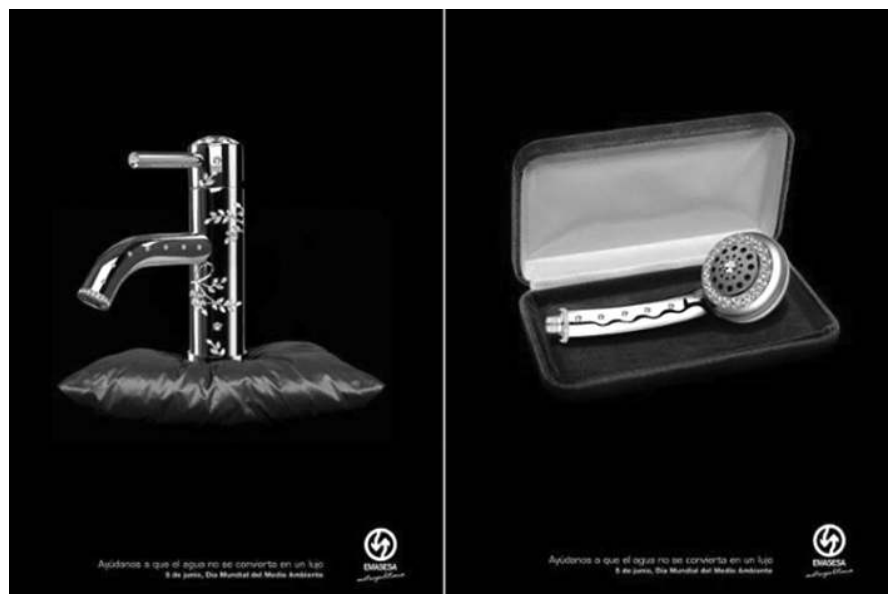

Source: EMASESA.

Pour Tim Moss, ce surdimensionnement a pour corollaire une aggravation des disparités spatiales, puisque, en raison des surcoûts engendrés et de la diminution des recettes pour les opérateurs, il favorise le développement de «cold spots4 » (Guy et Marvin, I996; Moss, 2008), qui seraient le versant sombre des «premium network spaces» (Graham et Marvin, 200I), ces espaces où l'offre en services urbains est de qualité supérieure. Si les travaux de Moss sont essentiels et d'une grande acuité pour bien saisir les processus à l'œuvre, ils se limitent à une analyse du phénomène de surdimensionnement, sans entrer pleinement dans les détails de l'après, à savoir de l'adaptation à (et de) ces réseaux surdimensionnés, ouvrant un champ d'exploration jusqu’ici non encore défriché. 


\section{QUELLES RECOMPOSITIONS À L'GUVRE DANS LE RÉSEAU À RÉGIME DÉCROISSANT? Le triple redimensionnement des réseaux}

Les réseaux d'eau et d'énergie ont longtemps constitué des rentes de fait pour les opérateurs, tant que la demande était en constante hausse. Sa diminution entraîne des changements inédits, puisque la source de revenus traditionnels se tarit progressivement. Le réseau à régime décroissant implique, pour en assurer la pérennité, un triple redimensionnement, qui est à la fois technique, territorial et économique. C’est ce que révèlent non seulement le détail des cas de Magdeburg et Séville mais aussi la littérature grise ou technique sur ces différents enjeux.

Il a fallu inventer de nouvelles manières de gérer les réseaux et de les adapter à de nouveaux régimes de consommation pour lesquels ils n'avaient pas été conçus. Trouver des solutions techniques garantissant la continuité du service et sa qualité tout en changeant le réseau: tel est le défi qui leur a été posé. L'opérateur de Magdeburg (Stadtwerk) a, de ce point de vue, poussé assez loin la logique intersectorielle qui est la sienne dès l'origine, en développant les capacités de ses employés à pouvoir intervenir sur les différents réseaux, aussi bien d'eau que d'énergie, ce qui est suffisamment rare pour être souligné. On observe à Magdeburg comme à Séville ou ailleurs une transformation des tuyaux, un changement dans la matérialité même du réseau, et des phénomènes inédits comme la fermeture de parties du réseau ou l'introduction de canalisations de petite taille pour s'adapter à une demande en berne, ou encore une recentralisation de la production. Dans la transformation infrastructurelle à l'œuvre, les ingénieurs et techniciens ont ainsi un rôle déterminant dans leur capacité à étendre la gamme des possibles dans leurs pratiques professionnelles.

Le redimensionnement est aussi territorial. La crise décrite est aussi une crise des économies d'échelle. Les réseaux voient, avec la baisse de la consommation et les surcoûts qu'elle engendre, s'éroder l'avantage de leur taille et les économies d'échelle associées. On observe ainsi des processus de changements territoriaux visant ce que les praticiens appellent «la bonne échelle» de gestion. Concrètement, la logique de décroissance de la demande amène une croissance des territoires desservis. Celle-ci semble assez aboutie à Magdeburg : 
dans le domaine électrique ou de l'eau, le Stadtwerk a réussi à racheter ou à contrôler des opérateurs des territoires proches, pour grossir et devenir un opérateur de dimension non plus locale mais régionale. Des réflexions similaires sont en cours à Séville, mais butent sur des questions politiques. Dans un contexte de diminution de la demande, les opérateurs viennent chercher une croissance de leur activité non plus dans leur marché stagnant ou déclinant, mais en prenant petit à petit le contrôle de territoires plus ou moins adjacents. Les opérateurs produisent ainsi une nouvelle échelle de gestion et donc de pouvoir et imposent de nouvelles formes de territorialités.

La transformation à l'œuvre est enfin celle du modèle d'activités. Les réseaux d'eau et d'énergie ont longtemps constitué des rentes de fait pour les opérateurs, tant que la demande était en constante hausse. Sa diminution entraîne des changements inédits, puisque la source de revenus traditionnels se tarit progressivement. Comme dans de nombreux secteurs d'activités faisant face à un marché déclinant, les opérateurs ont décidé de diversifier leurs sources de revenus. Deux directions majeures ont été retenues pour créer ces nouveaux leviers d'accumulation:

- un changement du lieu de production de la valeur dans les services urbains: c'est en ce sens que l'on doit comprendre le développement nourri de services en aval de la filière, à destination des usagers ;

- une production de valeur au-delà des seuls services urbains, qui s'inscrit dans une politique de diversification économique large.

Les baisses des consommations et des recettes afférentes ne semblent pas avoir sonné le glas des ambitions de croissance des firmes concernées, dans des cas comme ceux de Séville ou de Magdeburg où elles ont fait l'objet d’adaptations stratégiques de la part des opérateurs. Elles se sont davantage révélées comme l'accélérateur de nouvelles dynamiques qui correspondent finalement à la poursuite de la recherche de la croissance par d’autres moyens.

Le rôle des opérateurs, à travers ces changements de modèle d'activité, est ainsi modifié : ils deviennent plus que de simples pourvoyeurs de flux, en cherchant à être plus proches des usagers. 
La bifurcation infrastructurelle est finalement portée par cette transformation à la fois multiforme et inscrite dans la durée. Elle dit un changement des différentes composantes du réseau et également une modification de la trajectoire d'évolution de certains réseaux.

\section{COMMENT COMPLÉTER LES MODĖLES D'ÉVOLUTION DES RÉSEAUX?}

Cette trajectoire nouvelle permet sans doute de compléter les réflexions théoriques existantes sur le réseau et ses évolutions et de qualifier l'ampleur de la mutation à l'œuvre.

\section{I. LE RÉSEAU À RÉGIME DÉCROISSANT EST-IL UN POST-RÉSEAU?}

Les formes émergentes de réseaux produites par la diminution de la demande sont-elles solubles dans le post-réseau tel qu'il est construit par Olivier Coutard et Jonathan Rutherford (2013, 2016) ? Quelques faisceaux de raisons s'en rapprochent. Ces formes sont tout d'abord une illustration de la crise des grands réseaux techniques qui est au fondement de cette proposition théorique. Elles illustrent ainsi deux de ses composantes, à savoir la progressive émergence de systèmes composites, et la possible démultiplication de réseaux (Coutard et Rutherford, 20I3).

De façon assez claire, elles s'inscrivent aussi pleinement dans l'urbanisme post-réseau défini par le caractère non systématique d’une «convergence of sociotechnical systems around a networked configuration, model or paradigm» (Rutherford et Coutard, 2oı6, p. I). Cette absence de convergence, même si le paradigme du réseau ne disparaît pas complètement, se trouve au principe même de l'idée de bifurcation infrastructurelle, avec l'enchevêtrement des fermetures de pans du (grand) réseau et ses possibles élargissements.

Cependant, l'image de ces réseaux à la demande décroissante ne coïncide pas complètement avec toutes les transformations que le post-réseau est censé recouvrir. Si les différents cas étudiés empiriquement ou à travers la littérature indiquent bien une reconfiguration des espaces de solidarité liés aux systèmes sociotechniques, le passage, dans l'hypothèse du post-réseau, de 
logiques collectives à des logiques plus individuelles concernant les normes, pratiques et attentes vis-à-vis du service est moins évident, tout comme les nouvelles formes d’appropriation collective de l'infrastructure ${ }^{5}$, qui demeure l’apanage des opérateurs de réseau.

Les reconfigurations à l'œuvre dans ces réseaux à la demande décroissante les placeraient en fait dans une des catégories de post-réseau, celle de «l'hyper-réseau » (Barles et al., 2015). Cette figure est utilisée pour décrire les changements du réseau d'eau non potable de Paris longtemps délaissé, un temps menacé de fermeture définitive, et finalement récemment revitalisé afin de permettre une utilisation plus exhaustive des ressources disponibles (eaux de pluie, eaux grises, eaux d'exhaure) et du réseau. Les réagencements observés reprennent une logique assez proche, que ce soit via les changements d'échelle, ou les tentatives de mutualisations de ressources locales, ou en essayant parfois de boucler certains processus énergétiques. La crise des grands réseaux et l'émergence de nouveaux régimes de demande peuvent ainsi déboucher sur des alternatives qui renforcent la logique de réseau ${ }^{6}$ tout en modifiant certains aspects de son fonctionnement, en y intégrant notamment des processus de production moins linéaires et plus cycliques, à la lisière entre les avantages de la forme réticulaire et les bénéfices avancés par les tenants de solutions décentralisées et de petits réseaux. La trajectoire de l'hyper-réseau est non seulement une solution technique, mais également une réponse aux changements des logiques de gestion de l'offre et de la demande.

\subsection{LE RÉSEAU À RÉGIME DÉCROISSANT ET LA RECOMPOSITION DES LOGIQUES D'OFFRE ET DE LA DEMANDE}

L'un des changements fondamentaux apportés par le passage à des réseaux à la demande décroissante relève d'un bouleversement des logiques traditionnelles de gestion des opérateurs et de «l'idéal moderne de l'infrastructure » (Graham et Marvin, 200I). La gestion de l'offre et de la demande, selon qu'on

5 Voir Coutard et Rutherford, 2016 pour le détail de ces différents aspects.

6 Et qui pourraient trouver un écho dans les processus de production locale d'énergie autoconsommée ou réinjectée dans le réseau traditionnel. 
est dans un contexte de croissance ou de décroissance de la demande, diffère assez fortement. Les objectifs poursuivis par les opérateurs et les outils qu'ils mobilisent ne sont plus les mêmes. Comme le soulignait Moss (2008), en contexte de croissance, la gestion de l'offre a surtout consisté en une politique d'extension continue du réseau, et la gestion de la demande en une recherche d'écrêtage des pics et une amélioration de l'efficacité du réseau et de sa performance environnementale. Pour lui, la gestion de la demande en contexte de décroissance pousse à une stratégie opposée à celle de limitation des pics et de préservation des ressources, visant à inciter les usagers à utiliser plus d'eau ${ }^{7}$ via notamment des modifications des structures tarifaires. Cette optique ne correspond pas pleinement à ce qu'on peut observer : si certains responsables des opérateurs de Magdeburg promeuvent effectivement, dans leurs prises de parole publiques, l'idée qu'un meilleur service ne passe pas forcément par une moindre utilisation de l'eau, aucun outil tarifaire incitatif n'existe pour donner corps à cette ambition; du côté de Séville, on ne note également aucune transformation de ce type.

La diminution de la demande n'implique pas un renoncement à des objectifs de croissance et à une logique de l'offre, mais celle-ci se pare de nouveaux atours. La logique de gestion de l'offre dans un contexte de décroissance s'articule ainsi davantage autour des principes du redimensionnement et de l'interconnexion, là où la logique de gestion de l'offre dans un contexte de croissance de la demande repose sur la dynamique de l'accroissement spatial et volumétrique. On passe ainsi d'une logique d'extension à une logique de gestion de l'existant (Tableau I). La multiplication des expériences d'opérateurs confrontés à une décroissance de la demande permettrait sans doute de compléter un tableau certes encore embryonnaire, mais dont la vocation est aussi de montrer la transformation radicale à l'œuvre dans la gestion des réseaux selon les comportements de la demande, qu'on peut apparenter à un changement de paradigme. 
Tableau I. Gestion de l'offre et de la demande des grands réseaux techniques selon le régime d'évolution de la demande

\begin{tabular}{|l|c|c|}
\hline & $\begin{array}{c}\text { EN CONTEXTE DE CROISSANCE } \\
\text { DE LA DEMANDE }\end{array}$ & $\begin{array}{c}\text { EN CONTEXTE DE DÉCROISSANCE } \\
\text { DE LA DEMANDE }\end{array}$ \\
\hline $\begin{array}{l}\text { GESTION DE } \\
\text { L'OFFRE }\end{array}$ & $\begin{array}{l}\text { Logique du extend and supply: } \\
\text { - construction de grands ouvrages } \\
\text { - amplification de l'offre par } \\
\text { extension }\end{array}$ & $\begin{array}{c}\text { Logique de resizing: } \\
\text { - redimensionnement du réseau } \\
\text { - extension des zones desservies } \\
\text { pour maintenir - le niveau de } \\
\text { volumes consommés } \\
\text { - interconnexion des réseaux }\end{array}$ \\
\hline $\begin{array}{l}\text { GESTION } \\
\text { DE LA }\end{array}$ & Écrêtage des pics de demande & $\begin{array}{c}\text { Limitation des effets d'une vulnérabilité } \\
\text { accrue du réseau }\end{array}$ \\
DEMANDE & (exemple d'outils: tarif par bloc crois- & $\begin{array}{c}\text { sant, compteur individuel d'eau) } \\
\text { (exemple d'outils: flushing, recherche } \\
\text { de fuites, incitation ponctuelle à une } \\
\text { consommation accrue) }\end{array}$ \\
\hline
\end{tabular}

Source: élaboration de l'auteur.

\subsection{RÉSEAU À RÉGIME DÉCROISSANT ET MODÈLES D'ÉVOLUTIONS DU RÉSEAU}

L'émergence d'un réseau à régime décroissant n’implique pas de réinventer entièrement la notion même de réseau. La définition qu’en donnait Franck Scherrer dans sa thèse, en reprenant les travaux du groupe Réseaux, n’a pas fondamentalement varié : il s'agit toujours d'un agencement de lieux reliés les uns aux autres et entre lesquels circulent des flux (Scherrer, 1992). Les réseaux, pour reprendre sa formule, demeurent des objets «qui ont changé tout en restant les mêmes à l'échelle séculaire » (Scherrer, I992, p. IO). De la même façon, on peut toujours isoler, à la manière de Curien et Gensollen (I99I), les trois couches d'un réseau que sont les infrastructures (réseau-support), les services intermédiaires de contrôle (réseau de commande) et les services finaux d'utilisation (réseau-service).

En revanche, certaines des propriétés qui ont été associées aux réseaux techniques ont évolué et méritent une actualisation théorique. Si le réseau reste caractérisé par une certaine matérialité, une organisation dans l'espace de cette matérialité (avec une hiérarchisation de l'agencement technique) et un opérateur sur le long terme, la pérennité des territoires de référence que 
soulignait Scherrer (1992, p. 59) semble avoir vécu, ou tout du moins nécessiter quelques réajustements.

Le passage à un réseau à régime décroissant témoigne d’un changement de régime infrastructurel qu'il faut qualifier, tout en rappelant que les processus décrits ne concernent pas l'ensemble des opérateurs de réseaux, mais constituent une esquisse des modifications en cours. Cette transition vers un réseau à régime décroissant s'accompagne ainsi d'une transformation des paramètres constitutifs du réseau tels que Offner les définit (1993). La nouveauté du réseau à régime décroissant est de rebattre profondément les cartes de ces principes de fonctionnement du réseau, puisque ce ne sont plus un ou deux éléments qui changent, mais l'ensemble des composants du réseau qui sont modifiées (Figure 4). Les stratégies d’adaptation identifiées montrent ainsi :

- une transformation de la morphologie, puisqu'une partie des réseaux est mise hors service;

- un changement du réseau-support avec le redimensionnement des tuyaux;

- des changements dans les usages, dont la nécessité de créer des effets de chasse d'eau pour éviter la stagnation est un des exemples, tout comme les tentatives de bouclage énergétique ou de la récupération d'énergie issue de l'assainissement;

- un changement de mode de régulation et de modèle économique, qui voit notamment les modalités de gestion de l'offre et de la demande être passablement modifiées, et qui voit la production de valeur se déplacer vers l'aval de la filière, dans les services aux usagers, et parfois vers de nouveaux secteurs d’activité ;

- et enfin une transformation des territorialités, avec une production de nouvelles échelles de gestion et l'émergence de réseaux interconnectés à un niveau dépassant plus ou moins largement celui du territoire de référence antérieur. 
Figure 4. La transformation des modèles traditionnels des réseaux techniques

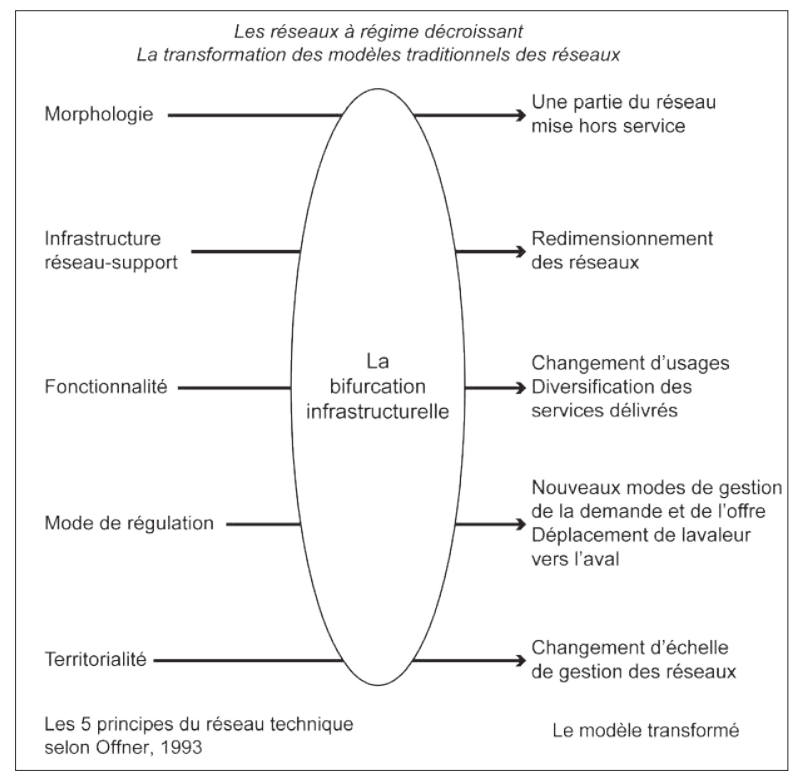

Source: élaboration de l'auteur.

Ces transformations multiples tracent les contours de nouvelles facettes du réseau et de ses modalités de fonctionnement. Elles n'épuisent cependant pas le modèle de réseau en train de se construire : le réseau à régime décroissant impose également des changements dans les cultures professionnelles et invite assez nettement à repenser les synergies entre réseaux pour compenser les pertes (volumétriques et financières) liées à ce nouveau contexte.

Ces mutations appellent un nouveau cadre analytique et conceptuel. Elles produisent un ensemble relativement inédit, un grand réseau revisité qui a su maintenir sa pérennité en devenant progressivement un hyper-réseau. Elles montrent en tout cas que les modèles de compréhension du réseau et de la firme locale d'infrastructures sont transformés en profondeur, et que cette transformation n'est pas qu'un ajustement à la marge (Naumann et Bernt, 2009). Cela correspond bien à une forme de changement de paradigme, de l'idéal de croissance continue à la gestion d'une demande plus faible dans une société à faible croissance ou sans croissance et qui se veut plus sobre énergétiquement. 


\section{BIBLIOGRAPHIE}

ALBIOL OMELLA C. et AGULLÓ AMORÓS F., 2014, «La reducción del consumo de agua en España: causas y tendencias», Aquae Papers, 6, Fundación Aquae.

BARLES S., COUTARD O. et GUILLERME A., 2016, «Beyond the Networked City, the Hyper-Networked City? Decline and Renaissance of the Parisian Non-Potable Water System (1820-2020)», in O. Coutard et J. Rutherford (dir.), Beyond the Networked City: Infrastructure Reconfigurations and Urban Change in the North and South, Londres, Routledge, p.46-64.

BARON M., CUNNINGHAM-SABOT E., GRASLAND C., RIVIÈRE D. et VAN HAMME G. (dir.), 2010, Villes et régions européennes en décroissance. Maintenir la cohésion territoriale, Paris, Éditions Lavoisier, Hermès.

BARRAQUÉ B., ISNARD L., MONTGINOUL M., RINAUDO J.-D. et J. SOURIAU., 201I, «Baisse des consommations d'eau potable et développement durable», Responsabilité et Environnement, 63, p. 102-108.

BEAUREGARD R., 2006, «The Radical Break in Late Twentieth Century Urbanization», Area, 38-2, p.218-220.

BIESBROEK G., SWART R. et VAN DER KNAAP W., 2009, «The MitigationAdaptation Dichotomy and the Role of Spatial Planning», Habitat international, 33, p. 230-237.

BOULEAU G., 20 II, «Introduction. Réseaux d'eau et services publics de gestion de l'eau», in G. Bouleau et L. Guérin-Schneider (dir.), Des tuyaux et des hommes, Versailles, Éditions Quæ , «Indisciplines», p. 13-22.

CALLON M. et FERRARY M., 2006, «Les réseaux sociaux à l'aune de la théorie de l'acteur-réseau», Sociologies pratiques, 2-13, p. 37-44.

COUTARD O., 2010a, «Services urbains: la fin des grands réseaux?», in O. Coutard et J.-P. Lévy (dir.), Écologies urbaines, Paris, Anthropos, p. I02-129. -, 2010b, «Focus. Services urbains, la fin d'un dogme», Annuels, p. 192-194.

- et RUTHERFORD J., 2013, «Vers l'essor de villes «post-réseaux »: infrastructures, innovation sociotechnique et transition urbaine en Europe» [en ligne]: $<$ http://www.enpc.fr/sites/default/files/coutard_rutherford_20l3_pnc.pdf>. 
- et RUTHERFORD J., 2016, «Beyond the networked city: an introduction», in Id., Beyond the Networked City: Infrastructure Reconfigurations and Urban Change in the North and South, Londres, Routledge, p. 7-24.

CURIEN N., 2000, Économie des réseaux, Paris, La Découverte.

- et GENSOLLEN M., 1991, «The Opening up of Networks: Planning and Competition in the Telecommunications Industry and other Public Utilities», Flux, I, p. $21-42$.

DJAMENT-TRAN G. et REGHEZZA-ZITT M., 20I2, Résiliences urbaines, les villes face aux catastrophes, Paris, Éditions du manuscrit.

EUZEN A., 2004, «Que se cache-t-il derrière les courbes de consommation d'eau? L'exemple de Paris», in D. Thevenot (dir.), 15e Journées scientifiques de l'environnement - Usages de l'eau: synergies et conflits, Créteil, JSE-2004, Journées Scientifiques de l'Environnement.

FLORENTIN D., 2015, Shrinking networks? Les nouveaux modèles économiques et territoriaux des firmes locales d'infrastructure face à la diminution de la consommation, thèse de doctorat, Université Paris-Est.

GARRISON W., 1990, «Networks: reminiscence and lessons», Flux, I, p. 5-12.

GRAHAM S. et MARVIN S., 200I, Splintering Urbanism. Networked Infrastructures, Technological Mobilities and the Urban Condition, Londres, Routledge.

GUY S. et MARVIN S., 1996, «Transforming Urban Infrastructure Provision-The Emerging Logic of Demand Side Management», Policy Studies, 17-2, p. I37- 147.

HANNEMANN C., 2003, «Schrumpfende Städte in Ostdeutschland. Ursachen und Folgen einer Stadtentwicklung ohne Wirtschaftswachstum », Politik und Zeitgeschichte, Bundeszentrale für politische Bildung, p. 16-23.

JACKSON T., 2005, Motivating Sustainable Consumption. A Review of evidence on consumer behaviour and behavioural change, Rapport pour le Sustainable Development Research Network, Guildford, University of Surrey.

JAGLIN S., 2005, Services d'eau en Afrique subsaharienne. La fragmentation urbaine en question, Paris, CNRS Éditions. 
KORNWACHS K., 1993, «Steuerung und Wachstum. Ein systemtheoretischer Blick auf grosse technische Systeme», in I. Braun et B. Joerges (dir.), Technik ohne Grenzen, Berlin, Suhrkamp Taschenbuch Wissenschaft, p. 4I0-445.

KLUGE T. et SCHEELE U., 2003, Transformationsprozesse in netzgebundenen Infrastruktursektoren. Neue Problemlagen und Regulationserfordernisse», netWORKS-Papers, I, Berlin.

KOZIOL M., 2008, «Räumliche Differenzierung der Infrastrukturversorgung. Chancen und Restriktionen im Rahmen des Stadtumbaus», in T. Moss, M. Naumann et M. Wissen (dir.). Infrastrukturnetze und Raumentwicklung. Zwischen Universalisierung und Differenzierung, Munich, Oekom Verlag, p. 173-I85.

LOOS A., 1898, Die Plumber, Vienne, Neue Freie Presse.

LOPEZ F., 20I4, Le Rêve d'une déconnexion. De la maison autonome à la cité autoénergétique, Paris, Éditions de la Villette.

MONSTADT J., 2009, «Conceptualizing the Political Ecology of Urban Infrastructures: Insights from Technology and Urban Studies», Environment and Planning A, 4I-8, p. 1924-1942.

MOSS T., 2008, «"Cold Spots" of Urban Infrastructure: Shrinking Processes in Eastern Germany and the Modern Infrastructural Ideal», IJURR, p. 436-45I.

NAUMANN M. et BERNT M., 2009, «When the Tap Stays Dry: Water Networks in Eastern Germany», Local Environment, 14-5, p. 46I-47I.

OFFNER J.-M., 1993, «Le développement des réseaux techniques: un modèle générique», Flux, 13, p. 11-18.

-, 1996, «Réseaux et Large Technical System: concepts complémentaires ou concurrents», Flux, 26, p. 17-30.

OWENS S., 1986, Energy, Planning and Urban Form, Cambridge, Pion Limited.

PFLIEGER G., 2009, L'Eau des villes. Aux sources des empires municipaux, Lausanne, Presses Polytechniques et Universitaires romandes.

SCHERRER F., 1992, L'Égout, patrimoine urbain. L'évolution dans la longue durée du réseau d'assainissement de Lyon, thèse de doctorat, UniversitéParis-Est Créteil. 
UKWIR (United Kingdom Water Industry Research), 2010, 21 $1^{\text {st }}$ Century Distribution Networks.

VILLARÍN CLAVERÍA M. C. et J. M. CAMARILLO NARANJO, 2012, «Factores que intervienen en el consumo doméstico de agua en las viviendas unifamiliares del municipio de Sevilla. Estudio a microescala», communication à la conférence ibérique de Lisbonne.

ZEPF M., SCHERRER F., VERDEIL E., ROTH H. et GAMBERINI J., 2008, Les Services urbains en réseau à l'épreuve des villes rétrécissantes: l'évolution des réseaux d'eau et d'assainissement à Berlin, rapport pour le Puca (Plan Urbanisme Construction Architecture). 